\title{
Modeling of fiber circumplacement around a hole using a streamline approach
}

\author{
Stefan Hartmann $^{\mathrm{a}^{*}}$ and Ali Kheiri Marghzar ${ }^{\mathrm{a}}$ \\ anstitute of Applied Mechanics, Clausthal University of Technology, Adolph-Roemer-Str. 2a, 38678 Clausthal-Zellerfeld, Germany \\ *Stefan Hartmann E-mail: stefan.hartmann@tu-clausthal.de
}

\author{
Article Info \\ Keywords: Borehole, Composite, \\ Streamline, Finite element analysis, \\ Transverse isotropy \\ 2010 AMS: 97Mxx, 74-XX, 74A20, \\ $74 B x x, 74 E 10$ \\ Received: 25 February 2018 \\ Accepted: 06 March 2018 \\ Available online: 11 March 2018
}

\begin{abstract}
The insertion of holes into laminates can be done by producing a fiber reinforced composite plate and, subsequently, drilling the borehole. Alternatively, we can bypass the fibers around the final hole before injecting the matrix material. In the first case, the spatial distribution of the axis of anisotropy, and the structural tensor concerned, are spatially constant. In the second case, i.e. the fiber circumplacement around the hole, a space-dependent anisotropy has to be considered. Instead of the common approach of defining region-wise constant fiber orientations, we propose a continuous formulation of fiber orientations using streamlines. To estimate the final stress and strain state for a unidirectional composite plate, three-dimensional finite element simulations are performed, where spatially constant transverse isotropy is compared to inhomogeneously distributed fiber orientation around the hole. It will turn out that the resulting stress states lead to both reduced stress amplitudes in loading direction as well as compressive strains in lateral direction. A detailed mathematical derivation of the basic equations accompanies the investigations.
\end{abstract}

\section{Introduction}

One possibility to produce laminates is to use one fiber direction. In this respect, woven fabrics are out the scope of the subsequent, first investigations. To compute a homogenized stress distribution, it is convenient to draw on linear elasticity for transverse isotropy for such kind of unidirectional laminates, see, for example, [1, 2], and, for a brief overview, [3]. These works go back to the fundamental contribution of [4]. In the field of small deformation, there exist a number of contributions, see, for example, [5, 6, 7, 8, 9]. There is a new area of applications of transverse isotropy, namely the field of biomechanics, since several soft tissues show this kind of anisotropic behavior, [10, 11, 12, 13, 14]. For particular descriptions, see [15, 16, 17]. Since there are only a very few analytical solutions (making sense for code verification purposes), numerical methods have to be chosen. Here, the finite element method is the most common approach. However, there might be also difficulties for low order elements. Regarding numerical difficulties, such as locking effects due to constraints in fiber direction under particular loading conditions, see [18, 19] and the literature cited therein. In our contribution fine meshes and quadratic shape functions are chosen to minimize numerical problems.

Fiber-reinforced plates must be connected to other structural elements, which is sometimes done using skews or rivets. Frequently, holes are drilled into the final plate so that some fibers are cut. To circumvent this, a suitable possibility is given by bypassing fibers around the hole in advance, i.e. during the production process.

Different methods have been developed for defining fiber orientation to investigate different issues of plates with a hole. Regarding stiffening laminates and to achieve variable stiffness in specimens, see [20,21]. Detailed research is available to optimize the fiber orientation near a hole, path optimization of laminated composite structures, and to find the optimal fiber angle distribution, see [22, 23, 24, 25]. [26, 27] study problems in buckling, failure, and vibration in laminates reinforced by curvilinear fibers. For more studies on stress, strain, fracture, and the influence of the thickness distribution in a ply with variable fiber angles, see [28, 29, 30, 31, 32, 33, 34]. Commonly, the works treat fiber orientation defined with piece-wise functions, see [24], or by element-wise formulations, see [27, 23].

In this study, fiber orientation is presented by using streamline function in fluid mechanics to obtain continuously distributed fiber orientations. Thus, we are interested in modeling both approaches using finite elements, where for the bypass approach a fiber orientation model 
using streamline theory around a circular cylinder is proposed. Finally, the results of the computations with uniform fiber orientation and the bypass-approach are compared.

The article is structured as follows: first, the constitutive model of transverse isotropy for the small strain case is derived from the large strain theory by geometrical linearization. Afterwards, we propose the streamline model to describe how fiber directions lie around a circular hole. Finally, finite element simulations are provided, where the stress and strain states for small strain applications are compared. The notation in use is defined in the following manner: geometrical vectors are symbolized by $\vec{a}$, second-order tensors $\mathbf{A}$ by bold-faced Roman letters, and calligraphic letters $\mathscr{A}$ define fourth order tensors. Furthermore, we introduce matrices by bold-faced Roman letters $\mathbf{A}$.

\section{Constitutive model of transverse isotropy}

In the following, we motivate the model of transverse isotropy for linear elasticity and a small strain theory. Afterwards, a model for a fiber orientation around a hole is proposed, which is based on the theory of streamlines.

\subsection{Basic equations}

Frequently, constitutive equations for transverse isotropy of an elastic material are modeled using a strain-energy function depending on the Green strain tensor $\mathbf{E}=(1 / 2)\left(\mathbf{F}^{T} \mathbf{F}-\mathbf{I}\right)$, or, alternatively, on the right Cauchy-Green tensor $\mathbf{C}=\mathbf{F}^{T} \mathbf{F}$, and a structural tensor $\mathbf{M}$ containing the information of the fiber orientation

$$
\tilde{\mathbf{T}}=\rho_{\mathrm{R}} \frac{\partial \psi(\mathbf{E}, \mathbf{M})}{\partial \mathbf{E}}=2 \rho_{\mathrm{R}} \frac{\partial \bar{\psi}(\mathbf{C}, \mathbf{M})}{\partial \mathbf{C}}
$$

$\mathbf{F}(\vec{X}, t)=\operatorname{Grad} \vec{\chi}_{\mathrm{R}}(\vec{X}, t)$ represents the deformation gradient of the motion $\vec{x}=\vec{\chi}_{\mathrm{R}}(\vec{X}, t)$, where the material point $\vec{X}$ occupies the spatial position $\vec{x}$ at time $t$. $\mathbf{I}=\delta_{i j} \vec{e}_{i} \otimes \vec{e}_{j}$ defines the second order identity tensor. In the case of transverse isotropy the structural tensor reads $\mathbf{M}=$ $\vec{a} \otimes \vec{a}$, where $\vec{a}(\vec{X})$ defines the orientation of the fiber direction with the property $\|\vec{a}\|=1$. $\tilde{\mathbf{T}}$ symbolizes the second Piola-Kirchhoff stress tensor, which is related to the (true) Cauchy stress tensor $\mathbf{T}$ by $\tilde{\mathbf{T}}=(\operatorname{det} \mathbf{F}) \mathbf{F}^{-1} \mathbf{T} \mathbf{F}^{-T} \cdot \rho_{\mathrm{R}}$ represents the density in reference configuration. The dependence of the strain energy function is not arbitrary but consists of particular number of invariants,

$$
\tilde{\mathbf{T}}=\rho_{\mathrm{R}} \frac{\partial \hat{\psi}\left(\mathrm{I}_{\mathbf{E}}, \mathrm{II}_{\mathbf{E}}, \mathrm{III}_{\mathbf{E}}, \mathrm{IV}_{\mathbf{E}}, \mathrm{V}_{\mathbf{E}}\right)}{\partial \mathbf{E}}=2 \rho_{\mathrm{R}} \frac{\partial \hat{\psi}\left(\mathrm{I}_{\mathbf{C}}, \mathrm{II}_{\mathbf{C}}, \mathrm{III}_{\mathbf{C}}, \mathrm{IV}_{\mathbf{C}}, \mathrm{V}_{\mathbf{C}}\right)}{\partial \mathbf{C}}
$$

which are defined by

$$
\begin{aligned}
& \mathrm{I}_{\mathbf{E}}=\operatorname{tr} \mathbf{E}, \mathrm{II}_{\mathbf{E}}=\operatorname{tr} \mathbf{E}^{2}, \mathrm{III}_{\mathbf{E}}=\operatorname{tr} \mathbf{E}^{3}, \mathrm{IV}_{\mathbf{E}}=\operatorname{tr}(\mathbf{E M})=\mathbf{E} \cdot \mathbf{M}, \mathrm{V}_{\mathbf{E}}=\operatorname{tr}\left(\mathbf{E}^{2} \mathbf{M}\right)=\mathbf{E}^{2} \cdot \mathbf{M}, \\
& \mathrm{I}_{\mathbf{C}}=\operatorname{tr} \mathbf{C}, \mathrm{II}_{\mathbf{C}}=\operatorname{tr} \mathbf{C}^{2}, \mathrm{III}_{\mathbf{C}}=\operatorname{tr} \mathbf{C}^{3}, \mathrm{IV}_{\mathbf{C}}=\operatorname{tr}(\mathbf{C M})=\mathbf{C} \cdot \mathbf{M}, \mathrm{V}_{\mathbf{C}}=\operatorname{tr}\left(\mathbf{C}^{2} \mathbf{M}\right)=\mathbf{C}^{2} \cdot \mathbf{M},
\end{aligned}
$$

see $[4,35]$. The invariants of the Green strain and right Cauchy-Green tensor are related by

$$
\mathrm{I}_{\mathbf{C}}=2 \mathrm{I}_{\mathbf{E}}+3, \quad \mathrm{II}_{\mathbf{C}}=4 \mathrm{II}_{\mathbf{E}}+4 \mathrm{I}_{\mathbf{E}}+3, \quad \mathrm{III}_{\mathbf{C}}=8 \mathrm{III}_{\mathbf{E}}+8 \mathrm{II}_{\mathbf{E}}+6 \mathrm{I}_{\mathbf{E}}+3, \quad \mathrm{IV}_{\mathbf{C}}=2 \mathrm{IV}_{\mathbf{E}}+1, \quad \mathrm{~V}_{\mathbf{C}}=4 \mathrm{~V}_{\mathbf{E}}+4 \mathrm{IV}_{\mathbf{E}}+1
$$

or vice versa

$$
\mathrm{I}_{\mathbf{E}}=\frac{1}{2}\left(\mathrm{I}_{\mathbf{C}}-3\right), \quad \mathrm{II}_{\mathbf{E}}=\frac{1}{4}\left(\mathrm{II}_{\mathbf{C}}-2 \mathrm{I}_{\mathbf{C}}+3\right), \quad \mathrm{III}_{\mathbf{E}}=\frac{1}{8}\left(\mathrm{III}_{\mathbf{C}}-2 \mathrm{II}_{\mathbf{C}}+\mathrm{I}_{\mathbf{C}}\right), \quad \mathrm{IV}_{\mathbf{E}}=\frac{1}{2}\left(\mathrm{IV}_{\mathbf{C}}-1\right), \quad \mathrm{V}_{\mathbf{E}}=\frac{1}{4}\left(\mathrm{~V}_{\mathbf{C}}-2 \mathrm{IV}_{\mathbf{C}}+1\right) .
$$

Since we are interested in formulating a constitutive model for small strains, we draw on the formulation using the Green strain tensor $\mathbf{E}$. Applying the chain rule on Eq.(2) 1 yields

$$
\tilde{\mathbf{T}}=\rho_{\mathrm{R}}\left(\frac{\partial \hat{\psi}}{\partial \mathrm{I}_{\mathbf{E}}} \mathbf{I}+\frac{\partial \hat{\psi}}{\partial \mathrm{II}_{\mathbf{E}}} \mathbf{E}+\frac{\partial \hat{\psi}}{\partial \mathrm{III}_{\mathbf{E}}} \mathbf{E}^{2}+\frac{\partial \hat{\psi}}{\partial \mathrm{IV}_{\mathbf{E}}} \mathbf{M}+\frac{\partial \hat{\psi}}{\partial \mathrm{V}_{\mathbf{E}}}(\mathbf{E M}+\mathbf{M E})\right)
$$

To obtain a linear elastic, small strain theory, which is fully appropriate in our application, several assumptions are introduced:

1. First, the Green strain tensor has to be linearized in view of the displacement gradient $\mathbf{H}=\operatorname{Grad} \vec{u}(\vec{X}, t)$ with $\vec{u}(\vec{X}, t)=\vec{\chi}(\vec{X}, t)-\vec{X}$,

$$
\mathbf{E}=\frac{1}{2}\left(\mathbf{H}+\mathbf{H}^{T}+\mathbf{H}^{T} \mathbf{H}\right)=\frac{1}{2}\left(\mathbf{H}+\mathbf{H}^{T}\right)+\mathscr{O}\left(\|\mathbf{H}\|^{2}\right),
$$

i.e. the quadratic term is omitted, see [36, 37].

2. Since we are interested in a theory of small displacements, it is assumed that there is no distinction between the spatial coordinates $\vec{x}$ and the coordinates in the reference configuration $\vec{X}$. Thus, the linearized Green strain tensor reads

$$
\mathbf{E}_{\mathrm{L}}(\vec{x}, t)=\frac{1}{2}\left(\operatorname{grad} \vec{u}(\vec{x}, t)+\operatorname{grad}^{T} \vec{u}(\vec{x}, t)\right) \approx \mathbf{E}
$$

implying that we have small displacements and small strains, i.e. $\vec{x} \approx \vec{X}, \vec{u}(\vec{X}, t)=\vec{u}(\vec{x}, t)$. There is no distinction of the configurations and all material properties are assigned to a spatial point $\vec{x}$. In the following, we omit the index $\mathrm{L}$ for brevity.

3. If we are interested in a linear theory, i.e. the resulting stress state $\mathbf{T}$ depends linearly on the strain state $\mathbf{E}$, and the strain-energy function (5) must quadratically depend on the strain tensor $\mathbf{E}$. This is given by

$$
\psi(\mathbf{E}, \mathbf{M})=\frac{\lambda}{2} \mathrm{I}_{\mathbf{E}}^{2}+\mu_{T} \mathrm{II}_{\mathbf{E}}+\alpha \mathrm{I}_{\mathbf{E}} \mathrm{IV}_{\mathbf{E}}+2\left(\mu_{L}-\mu_{T}\right) \mathrm{V}_{\mathbf{E}}+\frac{\beta}{2} \mathrm{IV}_{\mathbf{E}}^{2}
$$

There cannot be a dependence on the third invariant $\mathrm{III}_{\mathbf{E}}$, which automatically leads to a non-linear elastic material, see $[4,1,2]$. 
4. The stresses have the tendency to become equal for very small strains. Thus, we do not distinct between different stress measures, $\tilde{\mathbf{T}} \rightarrow \mathbf{T}$. Accordingly, we arrive at the final stress state applying Eq.(2) 1

$$
\mathbf{T}=\rho_{\mathrm{R}}\left(\left(\lambda \mathrm{I}_{\mathbf{E}}+\alpha \mathrm{IV}_{\mathbf{E}}\right) \mathbf{I}+2 \mu_{T} \mathbf{E}+\left(\alpha \mathrm{I}_{\mathbf{E}}+\beta \mathrm{IV}_{\mathbf{E}}\right) \mathbf{M}+2\left(\mu_{L}-\mu_{T}\right)(\mathbf{E M}+\mathbf{M E})\right)
$$

5. Frequently, the product of the density in the reference configuration with the material parameters is omitted so that the density is not visible in the final expression, i.e. we define

$$
\lambda \leftarrow \rho_{\mathrm{R}} \lambda, \quad \mu_{T} \leftarrow \rho_{\mathrm{R}} \mu_{T}, \quad \mu_{L} \leftarrow \rho_{\mathrm{R}} \mu_{L}, \quad \alpha \leftarrow \rho_{\mathrm{R}} \alpha, \quad \beta \leftarrow \rho_{\mathrm{R}} \beta
$$

Using all these assumptions lead to a particular expression, where elasticity relation (9) can be expressed by a fourth order elasticity tensor $\mathscr{C}$. To show this, we need the relations

$$
(\mathbf{B} \cdot \mathbf{C}) \mathbf{A}=(\mathbf{A} \otimes \mathbf{B}) \mathbf{C}, \quad \mathbf{A} \mathbf{C B} \mathbf{B}^{T}=[\mathbf{A} \otimes \mathbf{B}]^{T_{23}} \mathbf{C},
$$

see, for example, $[38,39,35]$, yielding

$$
\mathbf{T}=\mathscr{C} \mathbf{E}
$$

with

$$
\mathscr{C}=\lambda \mathbf{I} \otimes \mathbf{I}+2 \mu_{T} \mathscr{I}+\alpha[\mathbf{I} \otimes \mathbf{M}+\mathbf{M} \otimes \mathbf{I}]+\beta \mathbf{M} \otimes \mathbf{M}+2\left(\mu_{L}-\mu_{T}\right)[\mathbf{I} \otimes \mathbf{M}+\mathbf{M} \otimes \mathbf{I}]^{T_{23}} .
$$

$\mathscr{I}=[\mathbf{I} \otimes \mathbf{I}]^{T_{23}}=\delta_{i k} \delta_{j l} \vec{e}_{i} \otimes \vec{e}_{j} \otimes \vec{e}_{k} \otimes \vec{e}_{l}$ is the fourth order identity tensor, $\mathbf{A}=\mathscr{I} \mathbf{A}$. The symbolic $\mathscr{A}^{T_{23}}$ implies the transposition of second and third index, i.e. for $\mathscr{A}=a_{i j k l} \vec{e}_{i} \otimes \vec{e}_{j} \otimes \vec{e}_{k} \otimes \vec{e}_{l}$ we obtain $\mathscr{A}^{T_{23}}=a_{i k j l} \vec{e}_{i} \otimes \vec{e}_{j} \otimes \vec{e}_{k} \otimes \vec{e}_{l}$. For $\vec{a}=\vec{e}_{1}$, the Voigt notation of Eq.(13) is compiled in Appendix B.

\subsection{Model of fiber orientation}

To obtain a function with continuously distributed fibers, which move around a circular hole in the vicinity of the hole, we lend ideas of streamlines in Fluid Mechanics. In [40] the streamlines are defined by

$$
\psi=U_{\infty} \sin \theta\left(r-\frac{R^{2}}{r}\right)-k \frac{\log r}{R}
$$

We set $k=0, a=\psi / U_{\infty}, x=r \cos \theta$, and $y=r \sin \theta$ to arrive at

$$
g(x, y, a)=y^{3}-a y^{2}+\left(x^{2}-R^{2}\right) y-a x^{2}=0
$$

representing a cubic polynomial in $y$. There are three solutions, two conjugate complex and one real. We are only interested in the real solution yielding

$$
\begin{array}{r}
\hat{y}(x, a)=\frac{1}{6}\left(2^{2 / 3} \sqrt[3]{2 a^{3}+\sqrt{\left(2 a^{3}+9 a R^{2}+18 a x^{2}\right)^{2}-4\left(a^{2}+3(R-x)(R+x)\right)^{3}}+9 a R^{2}+18 a x^{2}}+\right. \\
\left.\frac{2\left(a^{2}+3(R-x)(R+x)\right)}{\sqrt[3]{a^{3}+\frac{1}{2} \sqrt{\left(2 a^{3}+9 a R^{2}+18 a x^{2}\right)^{2}-4\left(a^{2}+3(R-x)(R+x)\right)^{3}}+\frac{9 a R^{2}}{2}+9 a x^{2}}}+2 a\right)
\end{array}
$$

In Fig. 1(b) the orientation lines are shown for different $a$. The arbitrary factor $a$ is adapted in such a manner that it has a geometrical meaning, which is discussed in the following. The function (16) should have the value $\hat{y}\left(x^{*}, a^{*}\right)=y^{*} \leadsto a^{*}$. This constraint is fulfilled for

$$
a^{*}=\frac{\left(x^{* 2}-R^{2}\right) y^{*}+y^{* 3}}{x^{* 2}+y^{* 2}} .
$$

This parameter can be inserted into Eq.(15),

$$
f(x, y):=g\left(x, y, a^{*}\right)
$$

which can be evaluated, see Fig. 1(b), with $x^{*}=x$ and $y^{*}=y$. Thus, continuously distributed orientations are achieved. To obtain the tangent vector at the orientation function, we have to differentiate the position vector

$$
\vec{r}=x \vec{e}_{x}+\hat{y}(x) \vec{e}_{y},
$$

with respect to the arc-length, which can be chosen in the case of functions equivalent to $x$. Instead of applying Eq.(16), we apply the chain rule to Eq.(18),

$$
f(x, \hat{y}(x))=0, \quad f_{, x}+f, \hat{y}^{\prime}(x)=0 \quad \rightarrow \quad \hat{y}^{\prime}(x)=-f,{ }_{x} / f_{, y},
$$

which is a much simpler expression than using Eq.(16). Here, we draw on the abbreviations $f, x:=\partial f(x, y) / \partial x$ and $f, y:=\partial f(x, y) / \partial y$. The tangent vector at the orientation function reads

$$
\vec{t}(x)=\frac{\partial \vec{r}}{\partial x}=\vec{e}_{x}+\hat{y}^{\prime}(x) \vec{e}_{y}=\vec{e}_{x}-f, x / f, \vec{e}_{y} .
$$



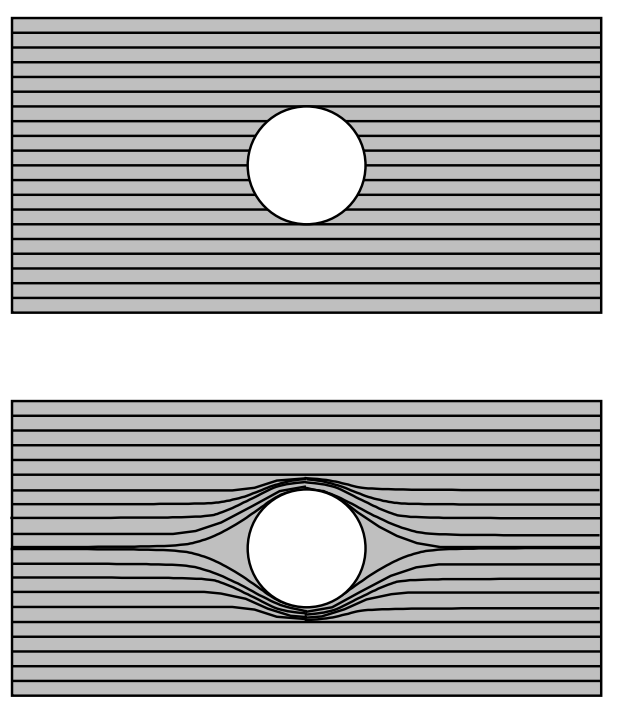

(a) Basic sketch of uniformly distributed fibers, where borehole is applied after production process, and circumplacement of fibers around the hole during the production process

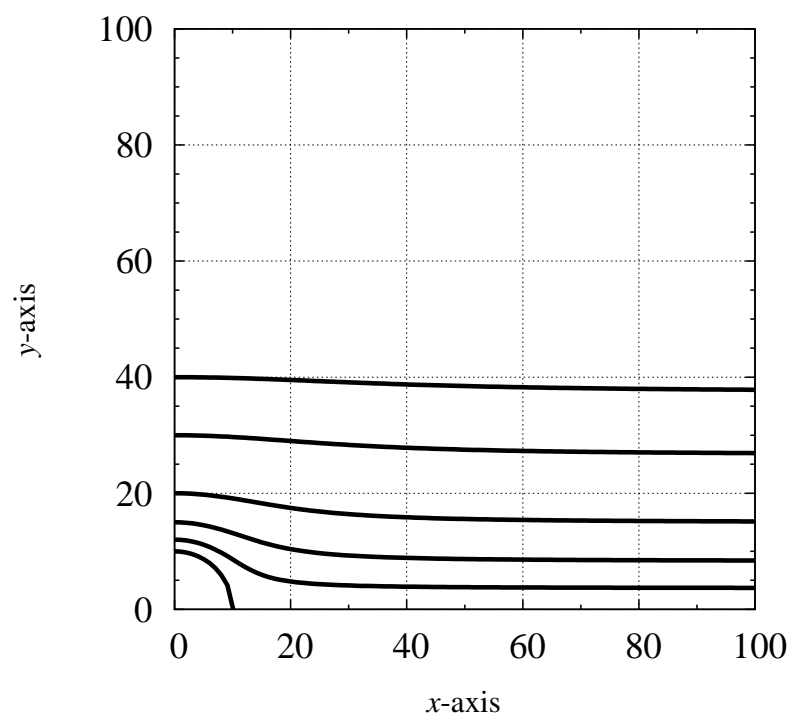

(b) Orientation lines using streamline functions

Figure 1: Orientation distribution of fibers

To obtain a unit vector at a point $x=x^{*}$ and $y=y^{*}$

$$
\vec{a}\left(x^{*}, y^{*}\right)=\left.\frac{\vec{t}\left(x^{*}\right)}{\left|\vec{t}\left(x^{*}\right)\right|}\right|_{x=x^{*}, y=y^{*}},
$$

with

$$
|\vec{t}(x)|=\sqrt{1+f,,_{x}^{2} f, y^{-2}}=\sqrt{f,,_{y}^{-2}} \sqrt{f,,_{x}^{2}+f,,_{y}^{2}} .
$$

Thus, the final unit tangent vector reads

$$
\vec{a}=\frac{1}{\sqrt{f,_{x}^{2}+f, 2}}\left(\sqrt{f,{ }_{y}^{2}} \vec{e}_{x}-f, x \frac{\sqrt{f,{ }_{y}^{2}}}{f, y} \vec{e}_{y}\right),
$$

which has to be evaluated at point $x=x^{*}$ and $y=y^{*}$. The superscript * can be omitted leading to the simple expressions

$$
f, x=2 x(y-a), \quad f, y=x^{2}-R^{2}-2 a y+3 y^{2}
$$

with

$$
a=\frac{\left(x^{2}-R^{2}\right) y+y^{3}}{x^{2}+y^{2}}
$$

\section{Finite element studies}

In the following, we compare two computations of a plate with a hole having a radius of $R=10 \mathrm{~mm}$. The plate is subjected to a displacement load of $u(100, y)=\bar{u}_{x}=0.01 \mathrm{~mm}$. It is meshed using 20-noded, isoparametric hexahedral elements with $(3 \times 3 \times 3)$ Gauss-points. For the geometry and the mesh, see Fig. 2. Here, only one-eighth of the plate is discretized due to symmetry conditions, see Fig. 2(b). The material parameters are lend from [4], $\lambda=5.64 \mathrm{Nmm}^{-2}, \mu_{T}=2.46 \mathrm{Nmm}^{-2}, \alpha=-1.27 \mathrm{Nmm}^{-2}, \beta=227.29 \mathrm{Nmm}^{-2}, \mu_{L}=5.66 \mathrm{Nmm}^{-2}$. In the first computation, we choose the spatially constant fiber orientation $\vec{a}=\vec{e}_{x}$ (homogeneous distribution of fiber orientation, in the following called uni-directional). In the second example, the fiber orientation of Eq.(24) is used, which is represented by the tangent vectors to the coordinate lines shown in Fig. 1(b). This is called bypass. Since this computation has the disadvantage to generate a singularity at point $(x, y, z)=(R, 0, z)$ generating arbitrary stresses close to this point - transition of circle to horizontal line -, we consider an improvement, see Fig. 3. A polynomial of third order is used to distinct a region, where no fibers are (below this polynomial), and the region using fiber orientation of Eq.(24). This computation, where the stiffness in $y$-direction is essentially weaker, is called bypass-reduced. The polynomial starts at point $(x, y)=(3 \sqrt{5}, 5)$ and ends at point $(x, y)=(15,0)$. Its slopes are the same as of the circle on the left, and zero on the right. Inside the small region, we take only the isotropic part of the elasticity relation (13) - first two terms of the elasticity tensor.

In the following, we study the stress and strain states in two computations (unidirectional and bypassed-reduced). We evaluate the stresses $\sigma_{x x}$ at $x=0$ and $z=1$ (vertical axis), $\sigma_{x x}(0, y, 1)$, see Fig. 4(a), and the stresses in vertical direction at the horizontal symmetry plane, $\sigma_{y y}(x, 0,1)$, see Fig. 4(b). The plots are generated using GiD, where interpolation schemes transfer Gauss-point information to nodal 


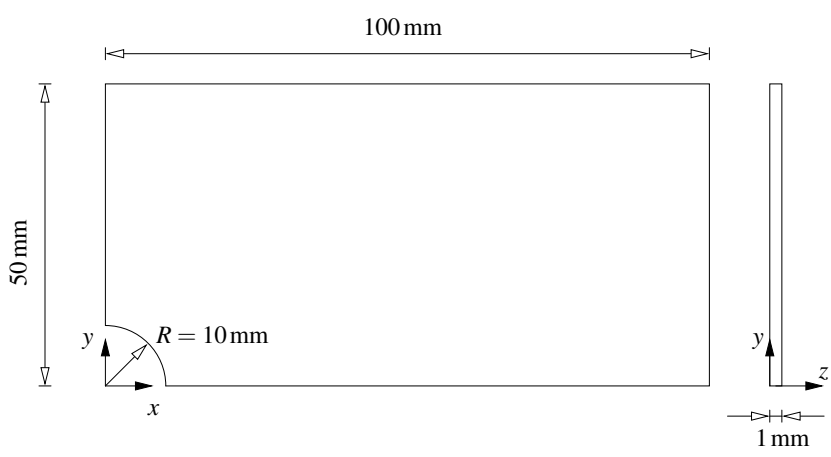

(a) Geometry of one-eighth of the specimen

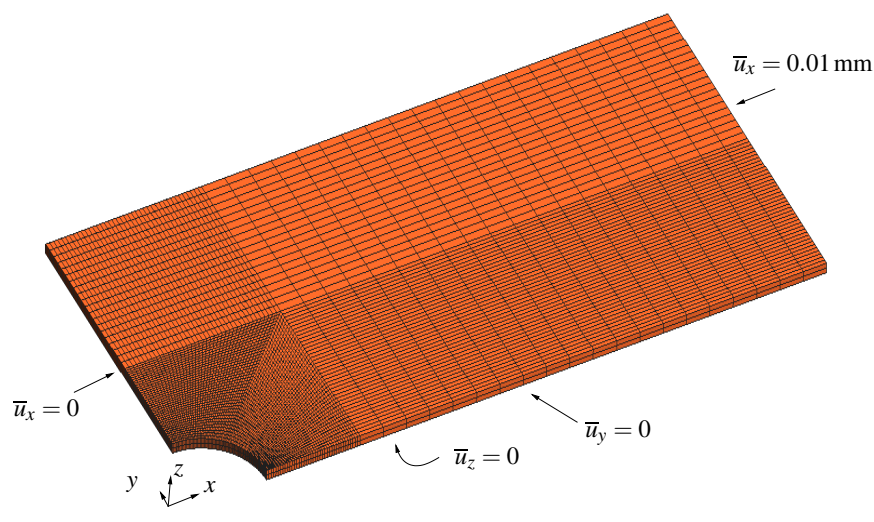

(b) Geometry (mesh and boundary conditions)

Figure 2: Geometry, mesh, and boundary conditions

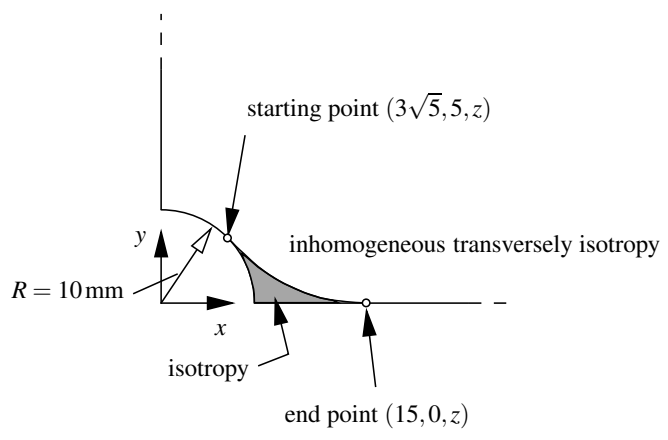

Figure 3: Region with purely isotropic material (bypass-reduced)

points. Obviously, the highest horizontal stresses are produced by a pure uniformly distributed fiber directions at point $(x, y)=(0, R)$. Very promising results are obtained for the "bypass-reduced"-simulation, where the stresses $\sigma_{x x}$ do not show such large amplitudes in this region (more than three times smaller).

Similar results are obtained for the strains, see Fig. 5. However, we obtain larger compressive strains $\varepsilon_{y y}$ for the bypass-reduced computation compared to the purely unidirectional reinforcement. However, negative strains are not so critical as tensile strains. Thus, promising results are obtained.

In Appendix $C$ the entire stress and strain distributions are assembled.

\section{Conclusions}

In this paper, we investigate the stress and strain distribution of unidirectional and inhomogeneous fiber directions around a hole, which is of interest in manufacturing processes. The circumplacement of fibers is modeled using streamline function to obtain the inhomogeneous fiber direction, which can be evaluated in finite element simulations of transversely isotropic material. The comparison shows a promising results, namely, that the stresses are essentially reduced around the hole. However, compressive strains, vertical to the loading directions, increase, which can be seen as not so dramatic then tensile strains. Further investigations and comparisons to experimental data have to be followed.

\section{A. Voigt-notation of transversal isotropy}

In the following, the tensorial notation is transferred into matrix notation so that no unnecessary computation should be performed. This is connected to the term Voigt-notation. Particularly, this is caused by the property of symmetry of the stress and strain tensor. Additionally, the scalar product in the principle of virtual displacements leads to a specific representation of the vector containing the independent strain tensor components leading to a symmetric elasticity matrix.

The (coefficients of the) components of the stress and strain tensor

$$
\mathbf{T}=\left[\begin{array}{ccc}
T_{11} & T_{12} & T_{31} \\
& T_{22} & T_{23} \\
\text { sym. } & & T_{33}
\end{array}\right] \vec{e}_{i} \otimes \vec{e}_{j}, \quad \mathbf{E}=\left[\begin{array}{ccc}
E_{11} & E_{12} & E_{31} \\
& E_{22} & E_{23} \\
\text { sym. } & & E_{33}
\end{array}\right] \vec{e}_{i} \otimes \vec{e}_{j}
$$

are assembled into column vectors

$$
\mathbf{T}^{T}=\left\{T_{11} T_{22} T_{33} T_{12} T_{23} T_{31}\right\}, \quad \tilde{\mathbf{E}}^{T}=\left\{E_{11} E_{22} E_{33} E_{12} E_{23} E_{31}\right\}
$$




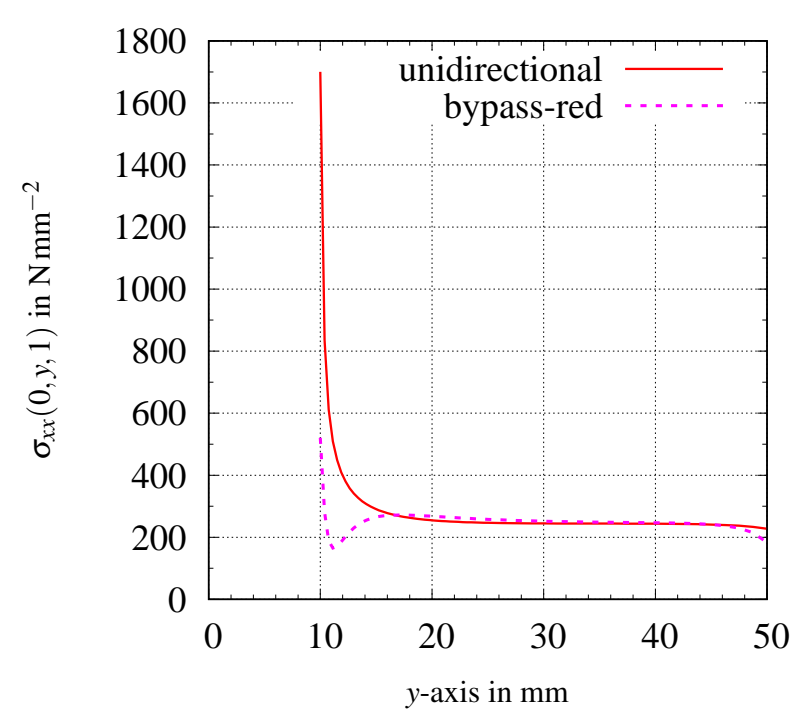

(a) Normal stresses $\sigma_{x x}$ in the vertical axis

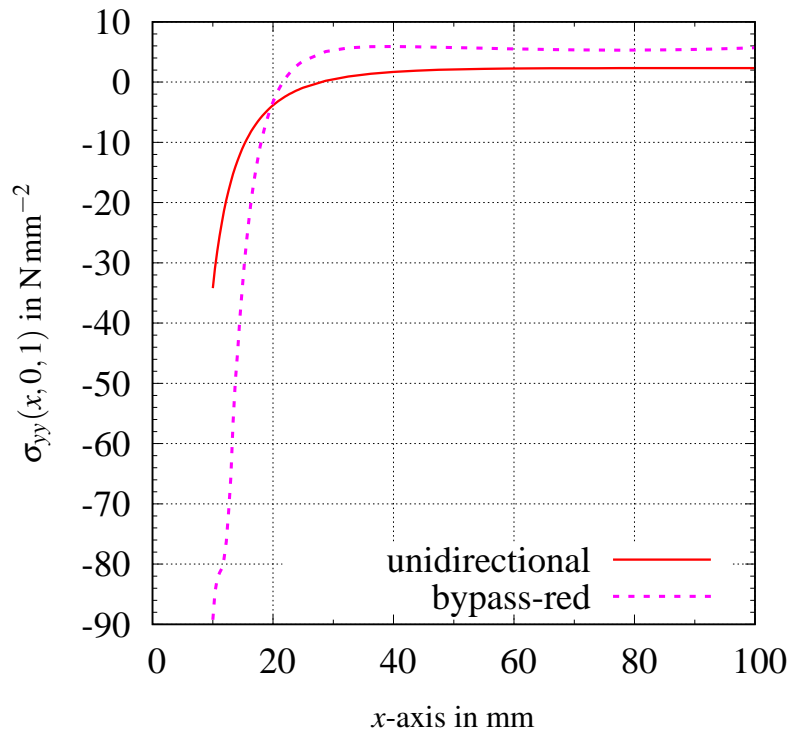

(b) Normal stresses $\sigma_{y y}$ in the horizontal axis

Figure 4: Stresses $\sigma_{x x}$ and $\sigma_{y y}$ at the vertical and the horizontal symmetry lines

To describe the influence of incorporating the symmetry properties into matrix formulation, we perform to steps: first, the tensorial quantities are transferred into $(9 \times 1)$ and $(9 \times 9)$-matrices, respectively. In this context, we treat the product (12) leading to

$$
\left\{\begin{array}{l}
T_{11} \\
T_{22} \\
T_{33} \\
T_{12} \\
T_{23} \\
T_{31} \\
T_{13} \\
T_{21} \\
T_{32}
\end{array}\right\}=\left[\begin{array}{lllllllll}
C_{1111} & C_{1122} & C_{1133} & C_{1112} & C_{1123} & C_{1131} & C_{1113} & C_{1121} & C_{1132} \\
C_{2211} & C_{2222} & C_{2233} & C_{2212} & C_{2223} & C_{2231} & C_{2213} & C_{2221} & C_{2232} \\
C_{3311} & C_{3322} & C_{3333} & C_{3312} & C_{3323} & C_{3331} & C_{3313} & C_{3321} & C_{3332} \\
C_{1211} & C_{1222} & C_{1233} & C_{1212} & C_{1223} & C_{1231} & C_{1213} & C_{1221} & C_{1232} \\
C_{2311} & C_{2322} & C_{2333} & C_{2312} & C_{2323} & C_{2331} & C_{2313} & C_{2321} & C_{2332} \\
C_{3111} & C_{3122} & C_{3133} & C_{3112} & C_{3123} & C_{3131} & C_{3113} & C_{3121} & C_{3132} \\
C_{1311} & C_{1322} & C_{1333} & C_{1312} & C_{1323} & C_{1331} & C_{1313} & C_{1321} & C_{1332} \\
C_{2111} & C_{2122} & C_{2133} & C_{2112} & C_{2123} & C_{2131} & C_{2113} & C_{2121} & C_{2132} \\
C_{3211} & C_{3222} & C_{3233} & C_{3212} & C_{3223} & C_{3231} & C_{3213} & C_{3221} & C_{3232}
\end{array}\right]\left\{\begin{array}{c}
E_{11} \\
E_{22} \\
E_{33} \\
E_{12} \\
E_{23} \\
E_{31} \\
E_{13} \\
E_{21} \\
E_{32}
\end{array}\right\}
$$

The symmetry of the stress tensor implies that the last three equations are the same as equations 4 to 6 :

$$
\left\{\begin{array}{l}
T_{11} \\
T_{22} \\
T_{33} \\
T_{12} \\
T_{23} \\
T_{31}
\end{array}\right\}=\left[\begin{array}{lllllllll}
C_{1111} & C_{1122} & C_{1133} & C_{1112} & C_{1123} & C_{1131} & C_{1113} & C_{1121} & C_{1132} \\
C_{2211} & C_{2222} & C_{2233} & C_{2212} & C_{2223} & C_{2231} & C_{2213} & C_{2221} & C_{2232} \\
C_{3311} & C_{3322} & C_{3333} & C_{3312} & C_{3323} & C_{3331} & C_{3313} & C_{3321} & C_{3332} \\
C_{1211} & C_{1222} & C_{1233} & C_{1212} & C_{1223} & C_{1231} & C_{1213} & C_{1221} & C_{1232} \\
C_{2311} & C_{2322} & C_{2333} & C_{2312} & C_{2323} & C_{2331} & C_{2313} & C_{2321} & C_{2332} \\
C_{3111} & C_{3122} & C_{3133} & C_{3112} & C_{3123} & C_{3131} & C_{3113} & C_{3121} & C_{3132}
\end{array}\right]\left\{\begin{array}{c}
E_{11} \\
E_{22} \\
E_{33} \\
E_{12} \\
E_{23} \\
E_{31} \\
E_{13} \\
E_{21} \\
E_{32}
\end{array}\right\}
$$

Now, we incorporate the symmetry of the strain tensor, $E_{12}=E_{21}, E_{23}=E_{32}, E_{31}=E_{13}$,

$$
\left\{\begin{array}{l}
T_{11} \\
T_{22} \\
T_{33} \\
T_{12} \\
T_{23} \\
T_{31}
\end{array}\right\}=\left[\begin{array}{llllll}
C_{1111} & C_{1122} & C_{1133} & C_{1112}+C_{1121} & C_{1123}+C_{1132} & C_{1131}+C_{1113} \\
C_{2211} & C_{2222} & C_{2233} & C_{2212}+C_{2221} & C_{2223}+C_{2232} & C_{2231}+C_{2213} \\
C_{3311} & C_{3322} & C_{3333} & C_{3312}+C_{3321} & C_{3323}+C_{3332} & C_{3331}+C_{3313} \\
C_{1211} & C_{1222} & C_{1233} & C_{1212}+C_{1221} & C_{1223}+C_{1232} & C_{1231}+C_{1213} \\
C_{2311} & C_{2322} & C_{2333} & C_{2312}+C_{2321} & C_{2323}+C_{2332} & C_{2331}+C_{2313} \\
C_{3111} & C_{3122} & C_{3133} & C_{3112}+C_{3121} & C_{3123}+C_{3132} & C_{3131}+C_{3113}
\end{array}\right]\left\{\begin{array}{c}
E_{11} \\
E_{22} \\
E_{33} \\
E_{12} \\
E_{23} \\
E_{31}
\end{array}\right\}
$$

In other words, we obtain the representation $\mathbf{T}=\tilde{\mathbf{C}} \tilde{\mathbf{E}}$, with $\mathbf{T} \in \mathbb{R}^{6}, \tilde{\mathbf{E}} \in \mathbb{R}^{6}$ and $\tilde{\mathbf{C}} \in \mathbb{R}^{6 \times 6}$. The arrangement of the coefficients of a fourth order tensor into the matrix $\tilde{\mathbf{C}}$ depends on the original calculation. For example, in the elasticity relation (13) we different products. First, we look at a product $\mathscr{C}=\mathbf{A} \otimes \mathbf{B}$, where $\mathbf{A}=\mathbf{A}^{T}$ and $\mathbf{B}=\mathbf{B}^{T}$ are symmetric tensors. The coefficients of $\mathscr{C}$ are given by $c_{i j k l}=a_{i j} b_{k l}$. In this case, the coefficient matrix (29) has the representation

$$
\mathbf{C}=\left[\begin{array}{llllll}
a_{11} b_{11} & a_{11} b_{22} & a_{11} b_{33} & 2 a_{11} b_{12} & 2 a_{11} b_{23} & 2 a_{11} b_{31} \\
a_{22} b_{11} & a_{22} b_{22} & a_{22} b_{33} & 2 a_{22} b_{12} & 2 a_{22} b_{23} & 2 a_{22} b_{31} \\
a_{33} b_{11} & a_{33} b_{22} & a_{33} b_{33} & 2 a_{33} b_{12} & 2 a_{33} b_{23} & 2 a_{33} b_{31} \\
a_{12} b_{11} & a_{12} b_{22} & a_{12} b_{33} & 2 a_{12} b_{12} & 2 a_{12} b_{23} & 2 a_{12} b_{31} \\
a_{23} b_{11} & a_{23} b_{22} & a_{23} b_{33} & 2 a_{23} b_{12} & 2 a_{23} b_{23} & 2 a_{23} b_{31} \\
a_{31} b_{11} & a_{31} b_{22} & a_{31} b_{33} & 2 a_{31} b_{12} & 2 a_{31} b_{23} & 2 a_{31} b_{31}
\end{array}\right]
$$

A fourth-order tensor having the transposition $T_{23}$ requires a more detailed consideration. We consider the product $T_{i j}=C_{i k j l} E_{k l}$ yielding 


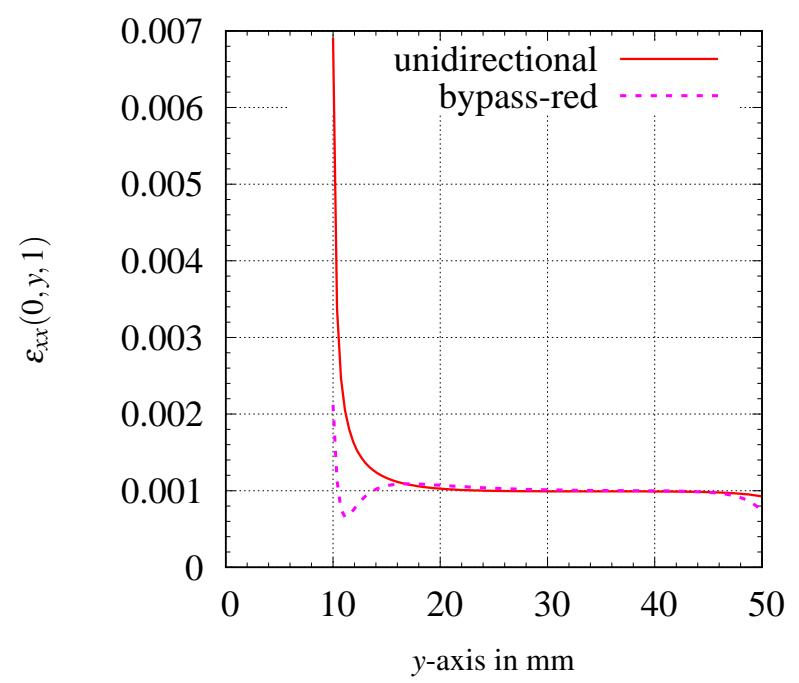

(a) Strain $\varepsilon_{x x}$ in the vertical axis

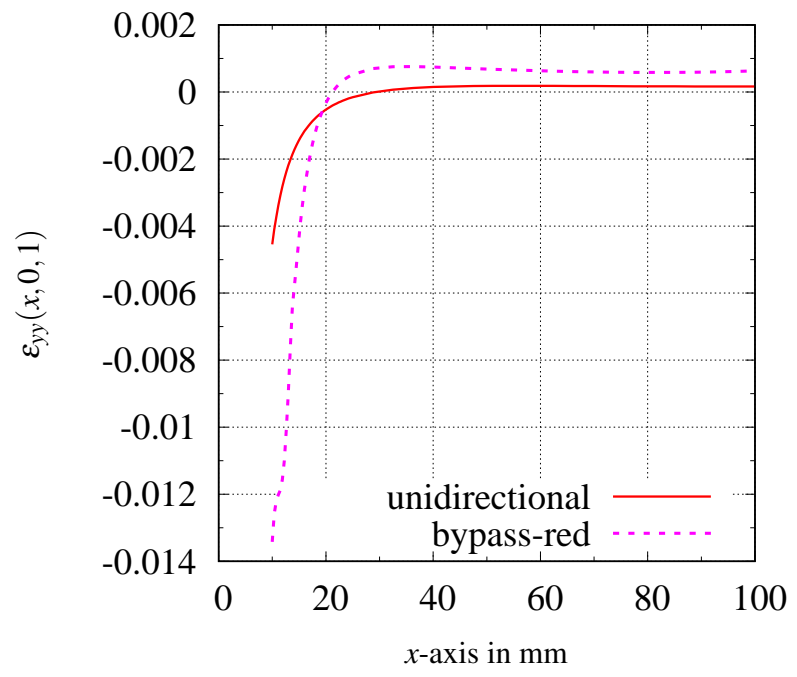

(b) Strain $\varepsilon_{y y}$ in the vertical axis

Figure 5: Strains $\varepsilon_{x x}$ and $\varepsilon_{y y}$ at the vertical and the horizontal symmetry lines

the $(9 \times 9)$-representation

$$
\left\{\begin{array}{l}
T_{11} \\
T_{22} \\
T_{33} \\
T_{12} \\
T_{23} \\
T_{31} \\
T_{13} \\
T_{21} \\
T_{32}
\end{array}\right\}=\left[\begin{array}{lllllllll}
C_{1111} & C_{1212} & C_{1313} & C_{1112} & C_{1213} & C_{1311} & C_{1113} & C_{1211} & C_{1312} \\
C_{2121} & C_{2222} & C_{2323} & C_{2122} & C_{2223} & C_{2321} & C_{2123} & C_{2221} & C_{2322} \\
C_{3131} & C_{3232} & C_{3333} & C_{3132} & C_{3233} & C_{3331} & C_{3133} & C_{3231} & C_{3332} \\
C_{1121} & C_{1222} & C_{1323} & C_{1122} & C_{1223} & C_{1321} & C_{1123} & C_{1221} & C_{1322} \\
C_{2131} & C_{2232} & C_{2333} & C_{2132} & C_{2233} & C_{2331} & C_{2133} & C_{2231} & C_{2332} \\
C_{3111} & C_{3212} & C_{3313} & C_{3112} & C_{3213} & C_{3311} & C_{3113} & C_{3211} & C_{3312} \\
C_{1131} & C_{1232} & C_{1333} & C_{1132} & C_{1233} & C_{1331} & C_{1133} & C_{1231} & C_{1332} \\
C_{2111} & C_{2212} & C_{2313} & C_{2112} & C_{2213} & C_{2311} & C_{2113} & C_{2211} & C_{2312} \\
C_{3121} & C_{3222} & C_{3323} & C_{3122} & C_{3223} & C_{3321} & C_{3123} & C_{3221} & C_{3322}
\end{array}\right]\left\{\begin{array}{c}
E_{11} \\
E_{22} \\
E_{33} \\
E_{12} \\
E_{23} \\
E_{31} \\
E_{13} \\
E_{21} \\
E_{32}
\end{array}\right\} .
$$

Obviously, in each column the indices 2 and 3 are exchanged, compared to representation (27). If we have the symmetries $T_{12}=T_{21}$, $T_{23}=T_{32}$, and $T_{31}=T_{13}$, the last three rows contain the same information as in rows 4 to 6 , and, accordingly, can be neglected. For the symmetries $E_{12}=E_{21}, E_{23}=E_{32}$, and $E_{31}=E_{13}$, we obtain

$$
\left[\begin{array}{llllll}
C_{1111} & C_{1212} & C_{1313} & C_{1112}+C_{1211} & C_{1213}+C_{1312} & C_{1311}+C_{1113} \\
C_{2121} & C_{2222} & C_{2323} & C_{2122}+C_{2221} & C_{2223}+C_{2322} & C_{2321}+C_{2123} \\
C_{3131} & C_{3232} & C_{3333} & C_{3132}+C_{3231} & C_{3233}+C_{3332} & C_{3331}+C_{3133} \\
C_{1121} & C_{1222} & C_{1323} & C_{1122}+C_{1221} & C_{1223}+C_{1322} & C_{1321}+C_{1123} \\
C_{2131} & C_{2232} & C_{2333} & C_{2132}+C_{2231} & C_{2233}+C_{2332} & C_{2331}+C_{2133} \\
C_{3111} & C_{3212} & C_{3313} & C_{3112}+C_{3211} & C_{3213}+C_{3312} & C_{3311}+C_{3113}
\end{array}\right] .
$$

If we apply these ideas to the tensors $\mathbf{I} \otimes \mathbf{I}, \mathbf{M} \otimes \mathbf{M}, \mathbf{I} \otimes \mathbf{M}+\mathbf{M} \otimes \mathbf{I}$ and $[\mathbf{I} \otimes \mathbf{M}+\mathbf{M} \otimes \mathbf{I}]^{T_{23}}$, we obtain the following representations:

$$
\begin{aligned}
& \mathbf{I} \otimes \mathbf{I} \longrightarrow\left[\begin{array}{cccccc}
1 & 1 & 1 & 0 & 0 & 0 \\
1 & 1 & 1 & 0 & 0 & 0 \\
1 & 1 & 1 & 0 & 0 & 0 \\
0 & 0 & 0 & 0 & 0 & 0 \\
0 & 0 & 0 & 0 & 0 & 0 \\
0 & 0 & 0 & 0 & 0 & 0
\end{array}\right] \quad \mathscr{I}=[\mathbf{I} \otimes \mathbf{I}]^{T_{23}} \longrightarrow\left[\begin{array}{cccccc}
1 & 0 & 0 & 0 & 0 & 0 \\
0 & 1 & 0 & 0 & 0 & 0 \\
0 & 0 & 1 & 0 & 0 & 0 \\
0 & 0 & 0 & 1 & 0 & 0 \\
0 & 0 & 0 & 0 & 1 & 0 \\
0 & 0 & 0 & 0 & 0 & 1
\end{array}\right] \\
& \mathscr{I}=[\mathbf{I} \otimes \mathbf{M}+\mathbf{M} \otimes \mathbf{I}]^{T_{23}} \longrightarrow\left[\begin{array}{cccccc}
2 m_{11} & 0 & 0 & 2 m_{12} & 0 & 2 m_{31} \\
0 & 2 m_{22} & 0 & 2 m_{12} & 2 m_{23} & 0 \\
0 & 0 & 2 m_{33} & 0 & 2 m_{23} & 2 m_{31} \\
m_{12} & m_{12} & 0 & m_{11}+m_{22} & m_{31} & m_{23} \\
0 & m_{23} & m_{23} & m_{31} & m_{22}+m_{33} & m_{12} \\
m_{31} & 0 & m_{31} & m_{23} & m_{12} & m_{11}+m_{33}
\end{array}\right] \\
& \mathbf{M} \otimes \mathbf{M} \longrightarrow\left[\begin{array}{cccccc}
m_{11}^{2} & m_{11} m_{22} & m_{11} m_{33} & 2 m_{11} m_{12} & 2 m_{11} m_{23} & 2 m_{11} m_{31} \\
m_{11} m_{22} & m_{22}^{2} & m_{22} m_{33} & 2 m_{12} m_{22} & 2 m_{22} m_{23} & 2 m_{22} m_{31} \\
m_{11} m_{33} & m_{22} m_{33}^{2} & m_{33} & 2 m_{12} m_{33} & 2 m_{23} m_{33} & 2 m_{31} m_{33} \\
m_{11} m_{12} & m_{12} m_{22} & m_{12} m_{33} & 2 m_{12}^{2} & 2 m_{12} m_{23} & 2 m_{12} m_{31} \\
m_{11} m_{23} & m_{22} m_{23} & m_{23} m_{33} & 2 m_{12} m_{23} & 2 m_{23}^{2} & 2 m_{23} m_{31} \\
m_{11} m_{31} & m_{22} m_{31} & m_{31} m_{33} & 2 m_{12} m_{31} & 2 m_{23} m_{31} & 2 m_{31}^{2}
\end{array}\right]
\end{aligned}
$$




$$
\mathbf{I} \otimes \mathbf{M}+\mathbf{M} \otimes \mathbf{I} \longrightarrow\left[\begin{array}{cccccc}
2 m_{11} & m_{11}+m_{22} & m_{11}+m_{33} & 2 m_{12} & 2 m_{23} & 2 m_{31} \\
m_{11}+m_{22} & 2 m_{22} & m_{22}+m_{33} & 2 m_{12} & 2 m_{23} & 2 m_{31} \\
m_{11}+m_{33} & m_{22}+m_{33} & 2 m_{33} & 2 m_{12} & 2 m_{23} & 2 m_{31} \\
m_{12} & m_{12} & m_{12} & 0 & 0 & 0 \\
m_{23} & m_{23} & m_{23} & 0 & 0 & 0 \\
m_{31} & m_{31} & m_{31} & 0 & 0 & 0
\end{array}\right]
$$

In finite elements, we have the scalar product of the stress tensor with the strain tensor (or with the virtual strain tensor). Since we would like to reduce the amount of function evaluations, we consider this scalar product

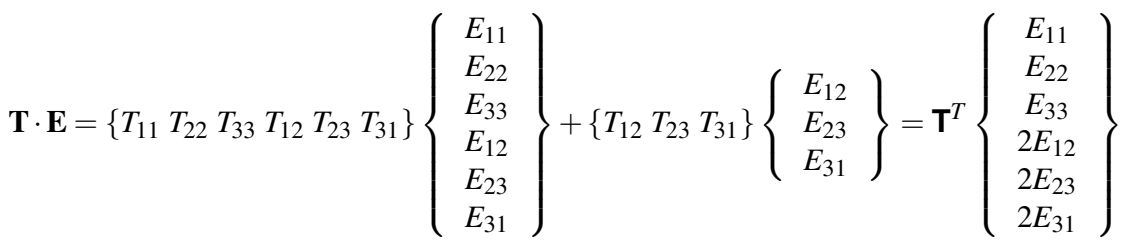

$$
\begin{aligned}
& =\mathbf{T}^{T} \mathbf{M} \tilde{\mathbf{E}}=\mathbf{T}^{T} \mathbf{E}
\end{aligned}
$$

with $\mathbf{M}=\operatorname{diag}(1,1,1,2,2,2) \in \mathbb{R}^{6 \times 6}$ and $\mathbf{E}^{T}=\left\{E_{11} E_{22} E_{33} 2 E_{12} 2 E_{23} 2 E_{31}\right\}$, i.e.

$$
\mathbf{E}=\mathbf{M} \tilde{\mathbf{E}} \quad \Longrightarrow \quad \tilde{\mathbf{E}}=\mathbf{M}^{-1} \mathbf{E} .
$$

Commonly, the "shear angles" $\gamma_{12}=2 E_{12}, \gamma_{23}=2 E_{23}, \gamma_{31}=2 E_{31}$ are introduced in Solid Mechanics implying that the product with 2 can be omitted. If we have the product $\mathbf{T}=\mathscr{C} \mathbf{E}$, i.e. $\mathbf{T}=\tilde{\mathbf{C}} \tilde{\mathbf{E}}=\mathbf{C E}$, the scalar product (37) reads:

$$
\mathscr{C} \mathbf{E} \cdot \mathbf{E}=\{\tilde{\mathbf{C}} \tilde{\mathbf{E}}\}^{T} \mathbf{E}=\left\{\tilde{\mathbf{C}} \mathbf{M}^{-1} \mathbf{E}\right\}^{T} \mathbf{E}=\{\mathbf{C E}\}^{T} \mathbf{E}=\mathbf{E}^{T} \mathbf{C E} .
$$

In other words, the last three columns of the coefficient matrices $\tilde{\mathbf{C}}$ must be multiplied with a factor $1 / 2$,

$$
\mathbf{C}=\tilde{\mathbf{C}} \mathbf{M}^{-1}
$$

yielding the side effect that all matrices (33) - (36) become symmetric.

\section{B. Case $\vec{a}=\vec{e}_{1}$}

Frequently, transversely isotropy is connected to the Voigt-notation, i.e. the $(6 \times 6)$-representation. In this case the orientation of the fibers is connected to one spatial coordinate direction, $\vec{a}=\vec{e}_{1}$ leading to the structural tensor $\mathbf{M}=\vec{a} \otimes \vec{a}=\vec{e}_{1} \otimes \vec{e}_{1}$, i.e. there is only the component $m_{11}=1$. All others are zero. In this case the matrix representation degenerates to

$$
\left\{\begin{array}{l}
T_{11} \\
T_{22} \\
T_{33} \\
T_{12} \\
T_{23} \\
T_{31}
\end{array}\right\}=\left[\begin{array}{cccccc}
\lambda+2 \alpha+2 \mu_{T}+4\left(\mu_{L}-\mu_{T}\right)+\beta & \lambda+\alpha & \lambda+\alpha & 0 & 0 & 0 \\
\lambda+\alpha & \lambda+2 \mu_{T} & \lambda & 0 & 0 & 0 \\
\lambda+\alpha & \lambda & \lambda+2 \mu_{T} & 0 & 0 & 0 \\
0 & 0 & 0 & \mu_{L} & 0 & 0 \\
0 & 0 & 0 & 0 & \mu_{T} & 0 \\
0 & 0 & 0 & 0 & 0 & \mu_{L}
\end{array}\right]\left\{\begin{array}{c}
E_{11} \\
E_{22} \\
E_{33} \\
\gamma_{12} \\
\gamma_{23} \\
\gamma_{31}
\end{array}\right\} .
$$

\section{Stress and strain distributions}

In the following, we compile the whole stress and strain distributions in Figs. 6 and 7.

\section{Acknowledgment}

We gratefully acknowledge the financial support provided by the government of Lower Saxony, Germany, for P2-project in the doctoral program "Self-organizing multifunctional structures for adaptive high performance light-weight constructions", which is located at the Campus Funktionswerkstoffe of the Clausthal University of Technology. Furthermore, we would like to thank Jithin Mohan for helpful discussions on streamlines and the support in preparing the computations. 

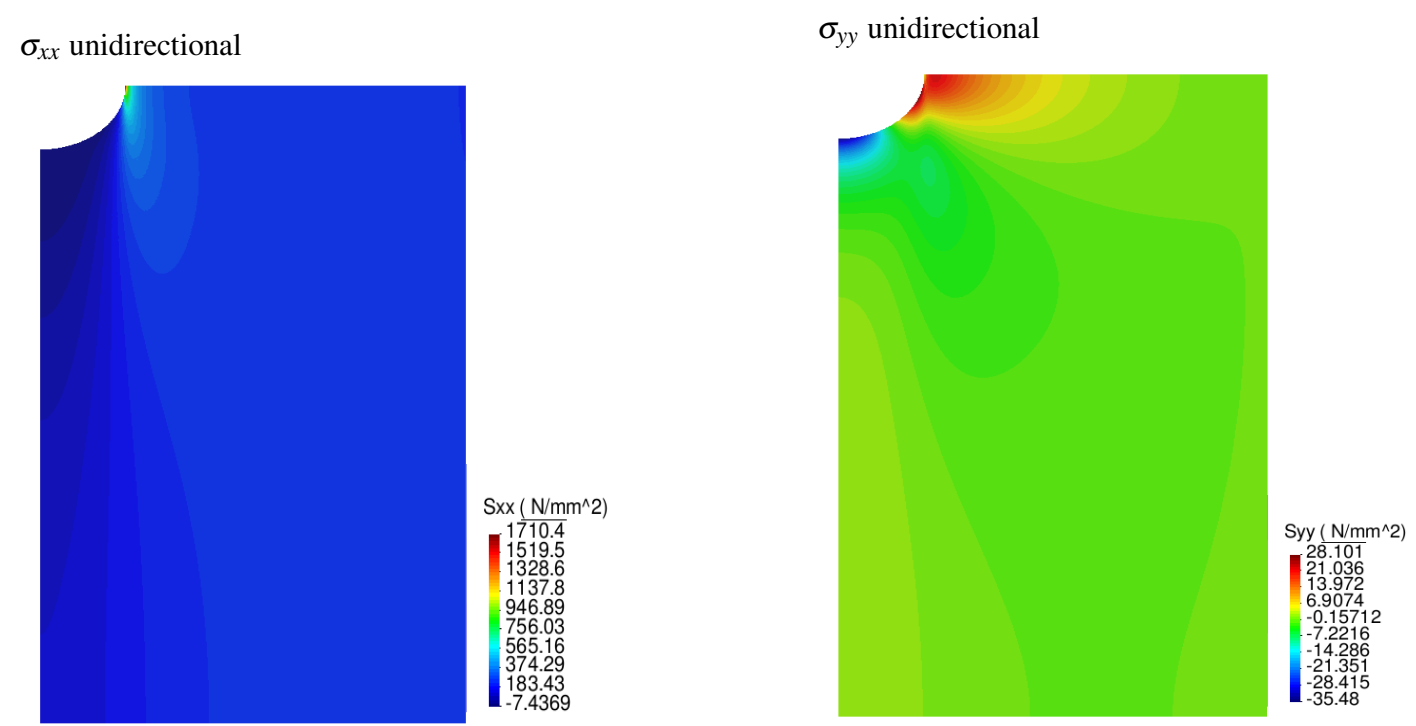

$\sigma_{x x}$ bypass-reduced

$\sigma_{y y}$ bypass-reduced
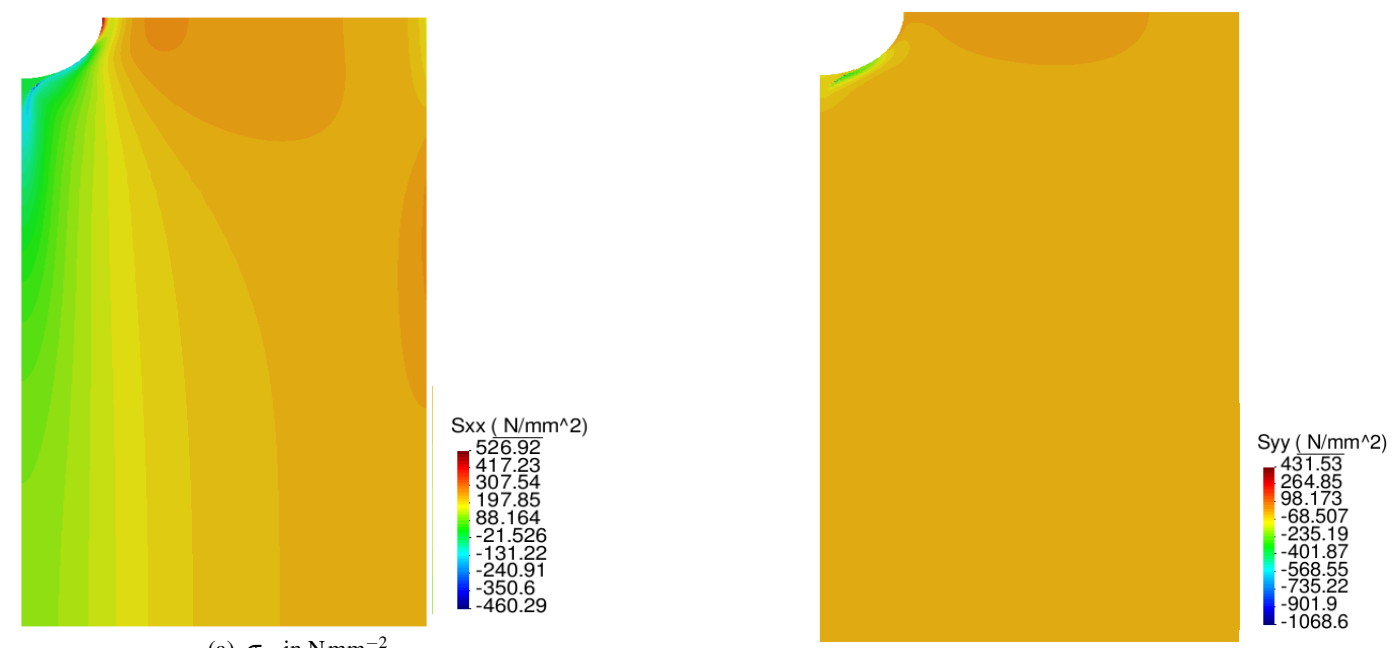

(b) $\sigma_{y y}$ in $\mathrm{Nmm}^{-2}$

Figure 6: Stress distributions 


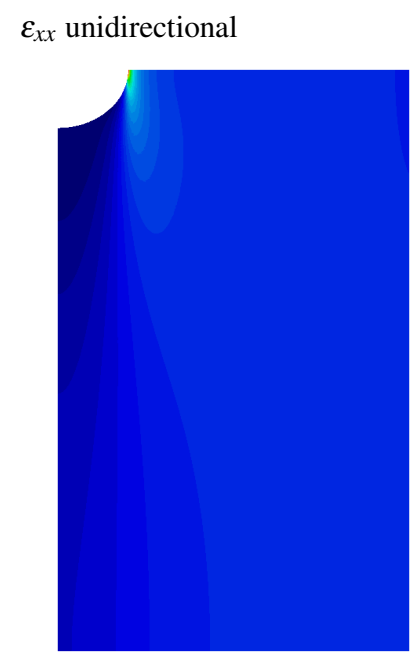

$\varepsilon_{x x}$ bypass-reduced

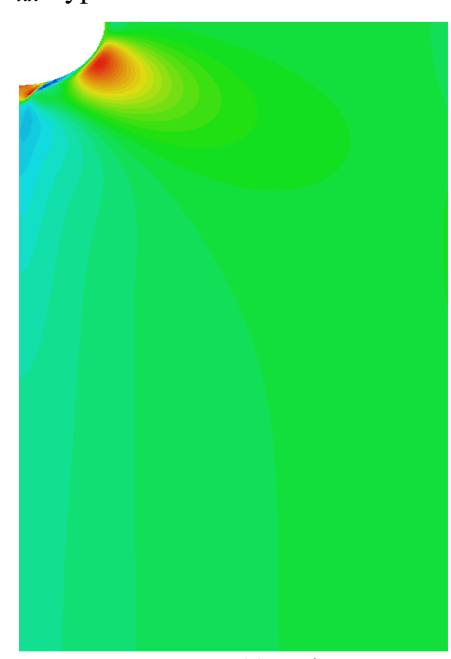

(a) strain $\varepsilon_{x x}$ $\varepsilon_{y y}$ unidirectional
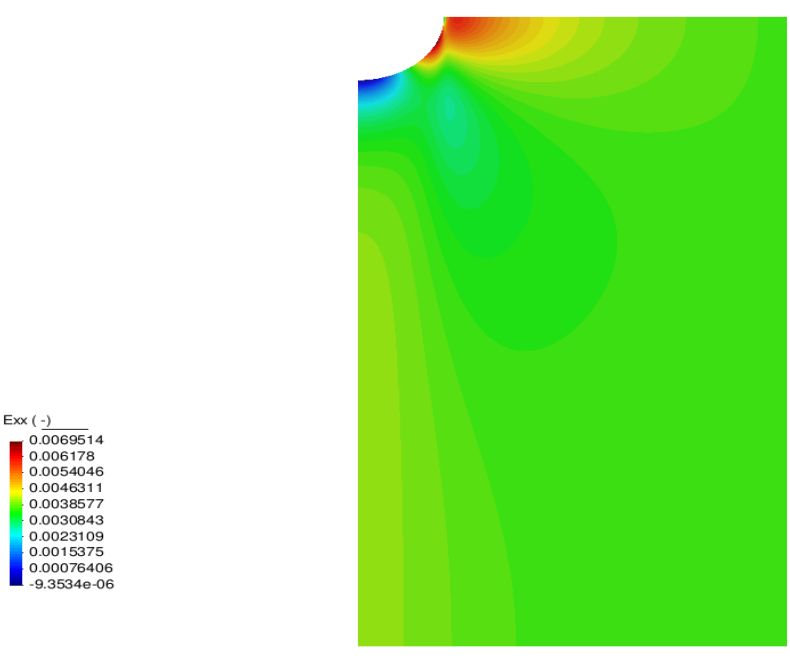

Evy (-)

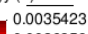

0.0026359

0.0017296

$-8.3105 \mathrm{e}-05$

$-0.00098945$

$-0.0018958$

$-0.0028021$

$-0.0037085$

$\varepsilon_{y y}$ bypass-reduced

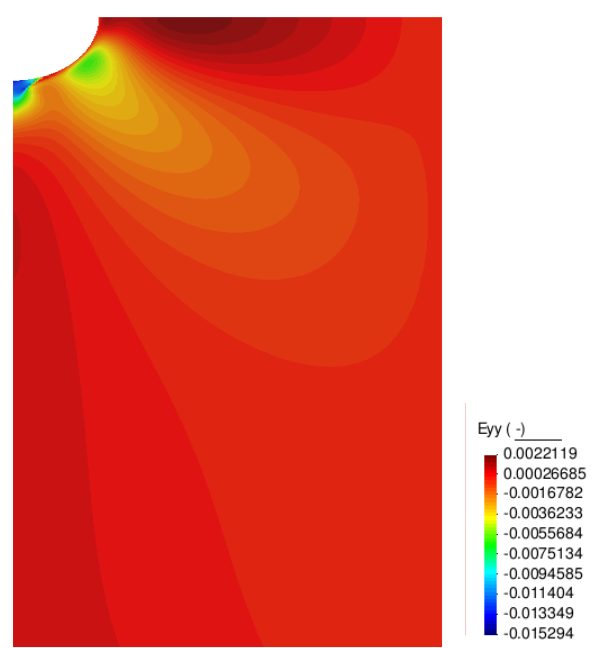

(b) strain $\varepsilon_{y y}$
$0 . \overline{0.0061767}$
0.0061767
0.0050988
0.0040208
0.0018649
0.0007897
$-0.0024469$

Figure 7: Strain distributions 


\section{References}

[1] M. Kaliske. Zur Theorie und Numerik von Strukturen aus Faserverbundmaterial. Mitteilung Nr. 49-99, Universität Hannover, 1999.

[2] M. Fiolka. Theorie und Numerik volumetrischer Schalenelemente zur Delaminationsanalyse von Faserverbundlaminaten. Report no. 2/2008, Institute of Mechanics, University of Kassel, 2008.

[3] M. Weise and A. Meyer. Grundgleichungen für transversal isotropes Materialverhalten. Preprint CSC/10-03, TU Chemnitz, Fakultät für Mathematik, Chemnitz (Germany), 2003.

[4] A. J. M. Spencer. Constitutive theory for strongly anisotropic solids. In A. J. M. Spencer, editor, Continuum theory of the mechanics of fibre-reinforced composites, number 282 in Courses and lectures - International Centre for Mechanical Sciences, pages 1-32. Springer-Verlag, Wien, 1984.

[5] B.D. Agarwal, L.J. Broutman, and K. Chandrashekhara. Analysis and Performance of Fiber Composites. Wiley, 2006.

[6] R.F. Gibson. Principles of Composite Material Mechanics, Third Edition. Mechanical Engineering. CRC Press, 2011.

[7] V.Z. Parton and B.A. Kudryavtsev. Engineering Mechanics of Composite Structures. CRC Press, Inc., 1993.

[8] J. Aboudi, S.M. Arnold, and B.A. Bednarcyk. Micromechanics of Composite Materials: A Generalized Multiscale Analysis Approach. Elsevier Science, 2012.

[9] J. Aboudi. Mechanics of Composite Materials: A Unified Micromechanical Approach. Studies in Applied Mechanics. Elsevier Science, 2013.

[10] J. A. Weiss, B. N. Maker, and S. Govindjee. Finite element implementation of incompressible, transversely isotropic hyperelasticity. Computer Methods in Applied Mechanics and Engineering 135 (1996), 107-128.

[11] J. Schröder and P. Neff. Invariant formulation of hyperelasticity transverse isotropy based on polyconvex free energy functions. International Journal of Solids and Structures 40 (2003), 401-445.

[12] J. Schröder, P. Neff, and D. Balzani. A variational approach for materially stable anisotropic hyperelasticity. International Journal of Solids and Structures 42 (2005), 4352-4371.

[13] M. Itskov and N. Aksel. A class of orthotropic and transversely isotropic hyperelastic constitutive models based on a polyconvex strain energy function. International Journal of Solids and Structures 41 (2004), 3833-3848.

[14] D. Balzani, P. Neff, J. Schröder, and G. A. Holzapfel. A polyconvex framework for soft biological tissues. adjustment to experimental data. International Journal of Solids and Structures 43 (2006), 6052-6070.

[15] G. A. Holzapfel, T. C. Gasser, and R. W. Ogden. A new constitutive framework for arterial wall mechanics and a comparative study of material models. Journal of Elasticity 61 (2000), 1-48.

[16] C. Sansour. On the physical assumptions underlying the volumetric-isochoric split and the case of anisotropy. European Journal of Mechanics - A/Solids 27 (2008), 28-39.

[17] A. Zdunek, W. Rachowicz, and T. Eriksson. A novel computational formulation for nearly incompressible and nearly inextensible finite hyperelasticity. Computer Methods in Applied Mechanics and Engineering 281 (2014), 220-249.

[18] P. Wriggers, J. Schröder, and F. Auricchio. Finite element formulations for large strain anisotropic material with inextensible fibers. Advanced Modeling and Simulation in Engineering Sciences, 3 (2016), 1-18.

[19] O. Sepahia, L. Radtke, S.E. Debus, and A. Düster. Anisotropic hierarchic solid finite elements for the simulation of passive-active arterial wall models. Computers \& Mathematics with Applications 74 (2017) 3058-3079.

[20] Gustavo Gonzalez Lozano, Ashutosh Tiwari, Christopher Turner, and Simon Astwood. A review on design for manufacture of variable stiffness composite laminates. Proceedings of the Institution of Mechanical Engineers, Part B: Journal of Engineering Manufacture 230(6) (2016), 981-992.

[21] X. J. Niu, T. Yang, Y. Du, and Z. Q. Xue. Tensile properties of variable stiffness composite laminates with circular holes based on potential flow functions. Archive of Applied Mechanics 86(9) (2016), 1551-1563.

[22] D. M. J. Peeters, S. Hesse, and M. M. Abdalla. Stacking sequence optimisation of variable stiffness laminates with manufacturing constraints. Composite Structures 125 (2015), 596-604.

[23] J. Huang and R. T. Haftka. Optimization of fiber orientations near a hole for increased load-carrying capacity of composite laminates. Structural and Multidisciplinary Optimization 30(5) (2005), 335-341.

[24] Yingdan Zhu, Jiancai Liu, Dong Liu, Haibing Xu, Chun Yan, Bin Huang, and David Hui. Fiber path optimization based on a family of curves in composite laminate with a center hole. Composites Part B: Engineering 111 (2017), 91-102.

[25] M. W. Hyer and R. F. Charette. Use of curvilinear fiber format in composite structure design. AIAA 125 (1991), $1011-1015$. 
[26] Pedro Ribeiro, Hamed Akhavan, Andrzej Teter, and Jerzy Warmiński. A review on the mechanical behaviour of curvilinear fibre composite laminated panels. Journal of Composite Materials 48(22) (2014), 2761-2777.

[27] M.W. Hyer and H.H. Lee. The use of curvilinear fiber format to improve buckling resistance of composite plates with central circular holes. Composite Structures 18(3) (1991), 239-261.

[28] R. E. Rowlands, I. M. Daniel, and J. B. Whiteside. Stress and failure analysis of a glass-epoxy composite plate with a circular hole. Experimental Mechanics 13(1) (1973), 31-37.

[29] A. W. Blom, M. M. Abdalla, and Z. Gürdal. Optimization of course locations in fiber-placed panels for general fiber angle distributions. Composites Science and Technology 70(4) (2010), 564-570.

[30] Lotfi Toubal, Moussa Karama, and Bernard Lorrain. Stress concentration in a circular hole in composite plate. Composite Structures 68(1) (2005), 31-36.

[31] A. V. Malakhov and A. N. Polilov. Construction of trajectories of the fibers which bypass a hole and their comparison with the structure of wood in the vicinity of a knot. Journal of Machinery Manufacture and Reliability 42(4) (2013), 306-311.

[32] A. V. Malakhov and A. N. Polilov. Design of composite structures reinforced curvilinear fibres using FEM. Composites Part A: Applied Science and Manufacturing 87 (2016), 23-28.

[33] A. Skordos, P. H. Chan, J. F. V. Vincent, and G. Jeronimidis. A novel strain sensor based on the campaniform sensillum of insects. Philosophical Transactions of the Royal Society of London A: Mathematical, Physical and Engineering Sciences 360, (2002), 239253.

[34] E. G. Koricho, A. Khomenko, T. Fristedt, and M. Haq. Innovative tailored fiber placement technique for enhanced damage resistance in notched composite laminate. Composite Structures 120 (2015), 378-385.

[35] M. Itskov. Tensor algebra and tensor analysis for engineers. Springer, Berlin, 2007.

[36] J. Casey. Approximate kinematical relations in plasticity. International Journal of Solids and Structures 21 (1985), 671-682.

[37] P. Haupt. Continuum Mechanics and Theory of Materials. Springer, Berlin, 2 edition, 2002.

[38] R. de Boer. Vektor- und Tensorrechnung für Ingenieure. Springer, Berlin, 1st edition, 1982.

[39] S. Hartmann. Finite-Elemente Berechnung inelastischer Kontinua. Interpretation als Algebro-Differentialgleichungssysteme. Habilitation, University of Kassel, Institute of Mechanics, 2003. Report No. 1/2003.

[40] F. M. White. Fluid Mechanics. McGraw-Hill, 2009. 